\title{
JComp: A Reuse-Driven Componentization Framework for Java Applications
}

\author{
Shimin Li and Ladan Tahvildari \\ Dept. of Electrical and Computer Engineering \\ University of Waterloo \\ Waterloo, Ontario, Canada, N2L 3G1 \\ \{s7li,ltahvild\}@uwaterloo.ca
}

\begin{abstract}
Program componentization has been proved to be an effective way to increase the speed and cost-effectiveness of reusing, maintaining, and understanding existing software systems. JComp is a componentization framework that supports semi-automatically extracting reusable components from an existing Java system and automatically transforming the existing system into a component-based system. Based on the automated class dominance analysis and domain knowledge, JComp introduces a novel technique to incrementally identify reusable components in a low cost but high precision way. Also, JComp provides a methodology to automate the transformation of existing object-oriented systems into component-based systems.
\end{abstract}

\section{Introduction}

An efficient and cost-effective software reuse process facilitates organizations to increase software productivity, quality, and reliability, and to decrease development costs and time. Component-Based Software Engineering (CBSE), which intends to build large software systems by integrating standard self-contained components, is a successful code reuse process [8]. However, one of the major issue in CBSE is the lack of a list of components with high reusability, mobility, and adaptability, despite the large amount of software that already exists in the portfolios of many software vendors. Therefore, seeking a cost-effective way to identify and extract reusable components from existing software systems is crucial for CBSE.

Caldiera and Basili [1] introduce the Care system to support identifying reusable components using user-defined reusability attribute model based on software metrics in the context of procedural paradigm. Etzkorn and Davis [2] present an approach for identifying reusable classes from object-oriented systems based on the understanding of comments and identifiers in the source code. Their tool CHRis uses natural-language techniques to help users to decide whether a class implements certain useful functionality. Shin and Kim [6] propose techniques for transforming an available objected-oriented design into a component-based design. Their techniques focus on formal model specification and transformation.

This research proposes a componentization framework that supports semi-automatically extracting reusable components from an existing Java system and automatically transforming the existing system into a component-based system. By applying class dominance analysis upon the class dependencies which are recovered through reverse engineering, and integrating domain knowledge into the extraction cycle, the framework incrementally identifies reusable components in a low cost but high precision manner. Furthermore, transforming the original system into a component-based system in order to improve the maintainability, the framework provides a methodology to automate the transformation process. JComp toolkit is the implementation of the proposed framework. It provides a highly integrated environment for: i) recovering and analyzing the structure of the system, ii) modeling the system, iii) incrementally identifying and extracting reusable components, and iv) performing source-to-source program transformation.

The remainder of the paper is organized as follows. Section 2 defines the terms used throughout of this research work. Section 3 presents the componentization approach. Section 4 describes the implementation of JComp toolkit. Finally, Section 5 gives the conclusion and outlines directions for further research.

\section{Terminology}

This section defines the terms that are used throughout of this research work.

A Reusable Component refers to a collection of functionally relating classes that provide a reusable service. A Program Entity is a class, interface, package, or a source file. 


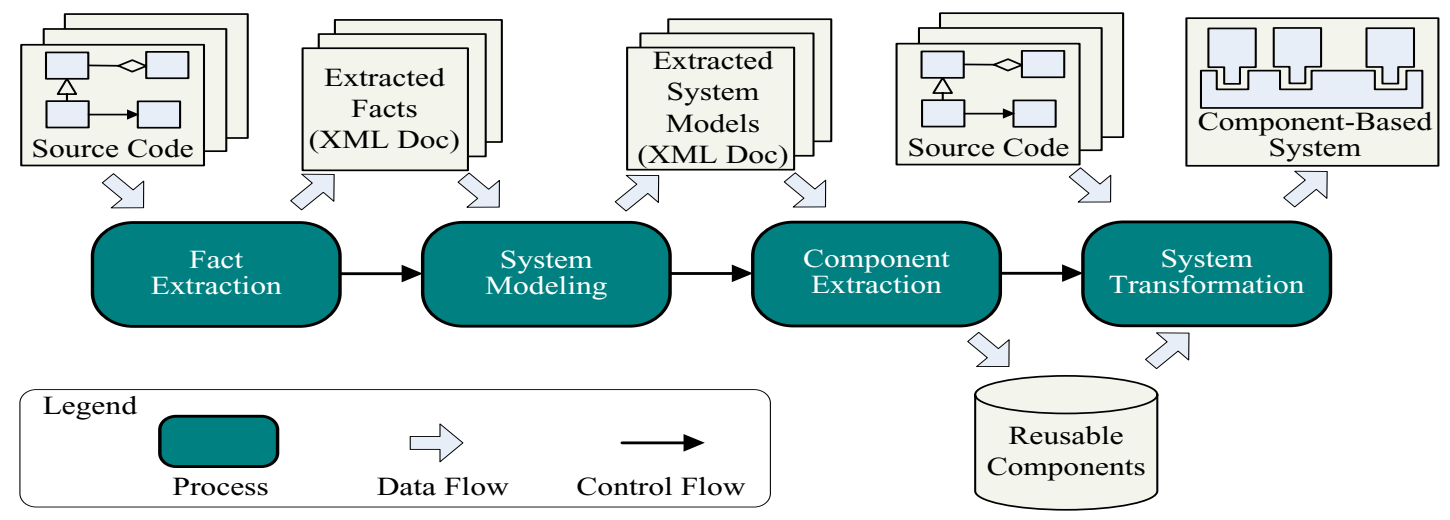

Figure 1. An Overview of the Reuse-Driven Componentization Framework.

The Base View (BView) of a system is the collection of facts of program entities while the Structural View (SView) consists of all relationships and dependency among program entities. The Class Relationship Graph $(C R G)$ is a directed graph that captures the various relationships among classes or interfaces such as inheritance, realization, association, aggregation, composition, and usage; while the Class Dependency Graph $(C D G)$ is an abstraction of the class relationship graph that only captures the dependency among them. Dominance is a relation between nodes in directed graphs [3]. A Dominance Tree (DTree) corresponding to a dependency graph is a tree whose nodes are the the nodes of the corresponding graph while its edges represent the dominance relationships between nodes. The dominance tree provides a high level description of the class dependency. A Reusability Attribute (RA) of a class is an indicator of reusability of the class, and in this context it refers to the complexity and coupling quality attributes, as well as the usage of the class in the existing system. A Class Clique is a set of classes that could provide a compound service. The Class-Cluster Dependency Graph (CCDG) is the modified class dependency graph by collapsing some nodes in the class dependency graph.

\section{Proposed Componentization Approach}

The proposed componentization approach is comprised of the following steps: i) extracting facts from source code, ii) modeling the system, iii) identifying and extracting reusable components, and iv) transforming the system. An overview of the reuse-driven componentization framework is illustrated in Figure 1. There are four processes defined in the framework and the following sections elaborate further on these processes.

\subsection{Fact Extraction Process}

The first automated process of the framework is to retrieve the facts of the Java program entities by applying reverse engineering. The extracted entity facts is essential for modeling the system and computing reusability attributes for each individual class in the next process. The results of this process are presented as XML documents and form the BView of the system.

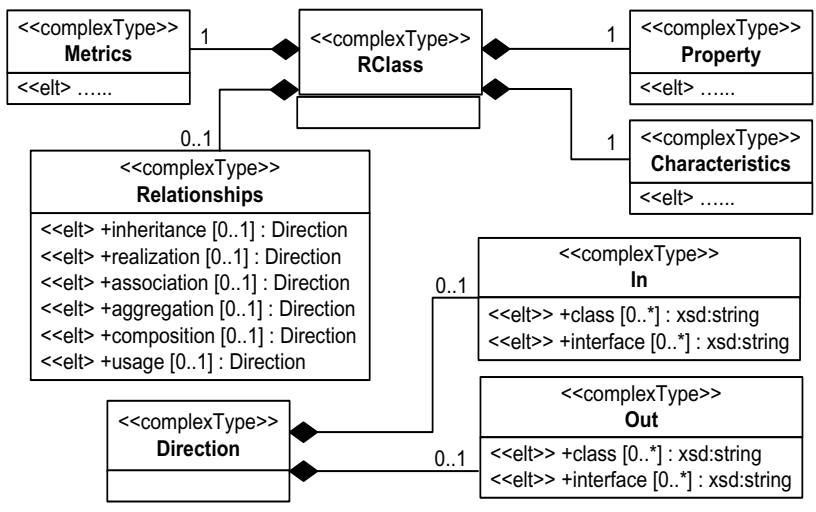

Figure 2. The UML Representation of XML Schema for Nodes in CRG.

\subsection{System Modeling Process}

The second automated process generates the CRG and CDG. Also reusability attributes for each class are computed and integrated into the graphs. The component identification and extraction tasks in the next process are performed upon the abstracted graph of these two graphs. The graphs are exported as XML documents and form the SView of the system. Figure 2 illustrates the relationships that JComp considers for its operation. 


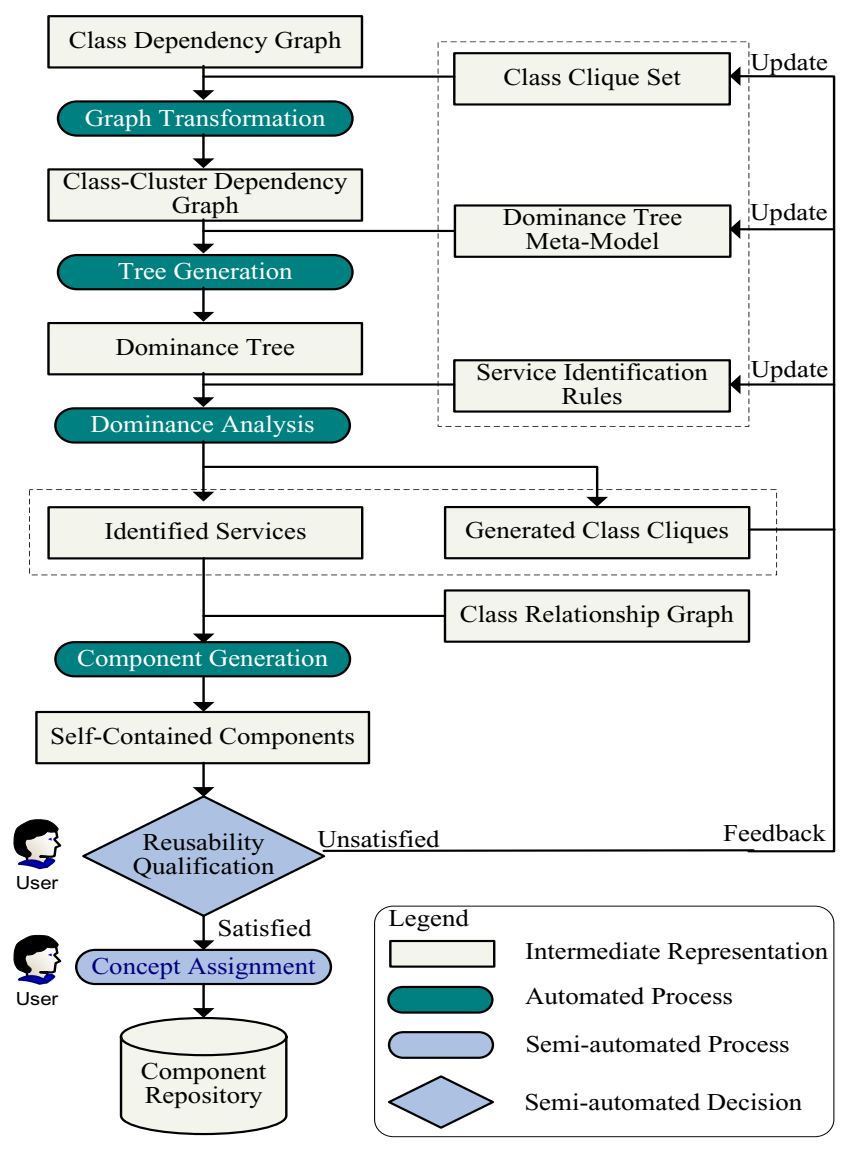

Figure 3. The Process of Identifying and Extracting Reusable Components in ObjectOriented Systems.

\subsection{Component Extraction Process}

The main prerequisite of the extracted component is to provide services which is useful for the further evolution of the software system and new systems. The framework presents a service-based component extraction technique that first identifies reusable services and then generates a component for each service. However, it is infeasible to decide in an automated way whether or not a service will be useful in a new system, since this decision is based on domain knowledge and the requirements of the new system. Therefore, the reusable component identification and extraction is a semi-automated process and needs proper feedback from the users. Figure 3 depicts the identification and extraction process. The component extraction is an iterative process and each iteration extracts a set of reusable components having the same level of granularity. The feedback in Figure 3 shows each iteration consists of five stages:

- Graph Transformation automatically generates the CCDG from CDG based on the class clique set that are suggested by the dominance analysis. The purpose of the generating CCDG in this stage is to identify services having greater granularity compared to the services identified in the previous iteration.

- Tree Generation automatically generates the DTree based on the dominance tree meta-model. The metamodel controls the shape of the generated DTree, while the shape is used to identify service for the next stage.

- Dominance Analysis automatically generates a set of services that have potential reuse from the dominance tree by applying service identification rules. The service identification rules determine which node or subtree in the tree may form a reusable service. Furthermore, the dominance analysis also suggests a set of class cliques which may be collapsed in the graph transformation stage of the next iteration to identify service with higher level of granularity.

- Component Generation automatically generates a selfcontained component for each identified service as a reusable candidate. This stage includes interface analysis and constituent extraction from the CRG.

- Reusability Qualification is a semi-automated process where the user performs reusability analysis on all extracted candidates and makes a decision on acceptable candidates. The feedback from the users updates the service identification rules, dominance tree metamodel, or class clique set, and repeat the extraction procedure until the number and the granularity level of accepted components are satisfied.

Once all reusable components are extracted, the last task of the extraction process is the Concept Assignment which also is a semi-automated task involving users. It includes naming and documenting the extracted components.

\subsection{System Transformation Process}

In order to transform the source system into a target component-based system, we propose a reference model for component-based systems. In this context, the reference model defines the hierarchical composition of the selfcontained components that are extracted from the source system. The composition among components can be achieved since the functionality of each component is selfcontained. The first task in the transformation process is to generate the component hierarchy. Then, for each component, the references to the classes within its inner components are detected and modified so that the modified references refer the interface of the corresponding component.

\section{JComp Implementation}

The proposed reuse-driven componentization framework has been implemented as a toolkit named JComp - Java 


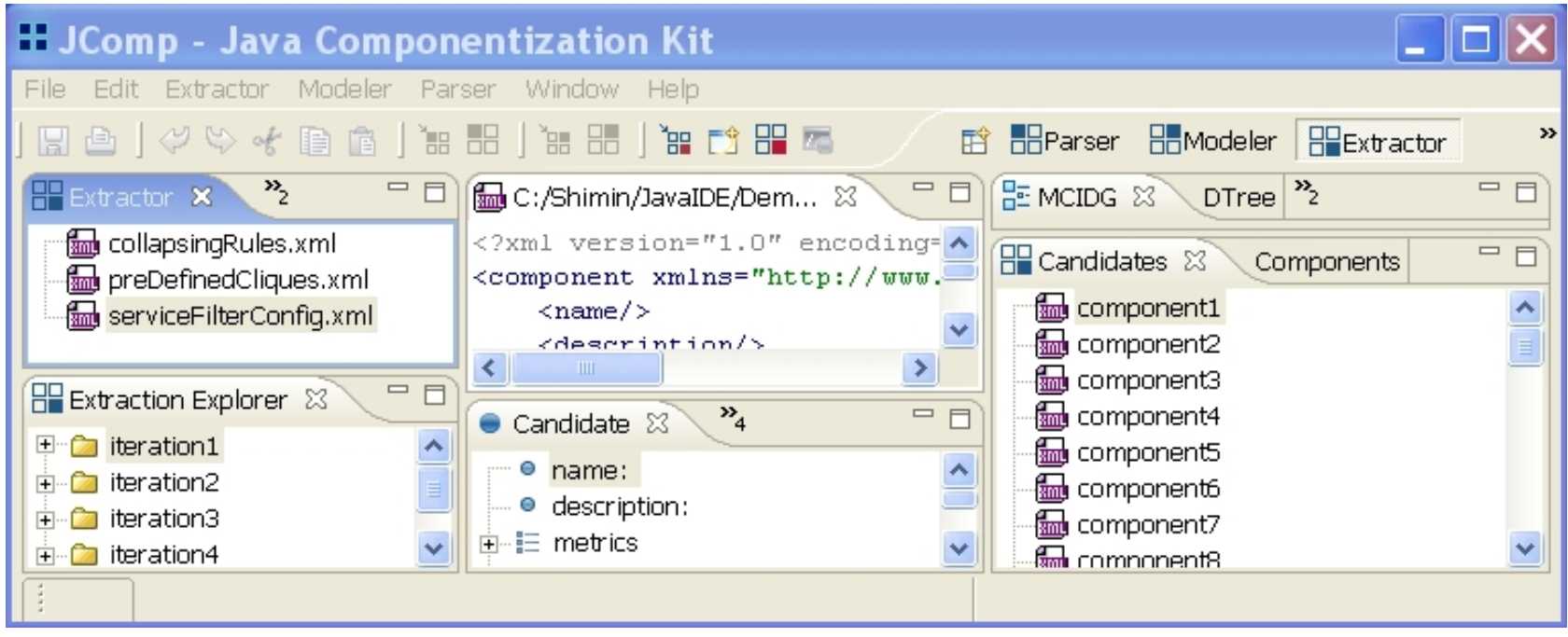

Figure 4. A Screenshot of the JComp Java Componentization Kit.

Componentization Kit. The toolkit consists of the following four components as illustrated in Figure 1:

- Java Source Code Parser: The parser is a JavaCCbased [7] parser. It automatically extracts facts for all program entities.

- Java System Modeler: The modeler automatically models the system and computes the reusability attributes for each class.

- Component Extractor: The extractor interacts with users to complete the extraction procedure discussed Section 3.3.

- Component-Based System Transformer: The transformer automatically transforms the source system into the component-based system taking the source code and extracted components as its inputs.

The JComp toolkit is implemented as an Eclipse Rich Client Platform (RCP) [5] in an integrated environment. Such a comprehensive environment integrates the parser, modeler, extractor and transformer components of JComp, and provides a flexible workspace for users to view various intermediate results and interact with the toolkit. Figure 4 shows a screenshot of JComp running as an Eclipse RCP application.

\section{Conclusions and Future Work}

In this paper, we presented a reuse-driven framework that supports semi-automatically extracting reusable components from an existing Java system and automatically transforming the existing system into a target component-based system. The framework incrementally identifies reusable components in a low cost but high precision manner. It also provides a methodology to automate the transformation process. Case studies, which we performed using JComp, show that the proposed framework provides an cost-effective way to componentize Java applications and the various system views (BView, SView, DTree) produced by such process help users to gain a program understanding of the application.

The next step is to apply the proposed framework on large Java systems to further identify its benefits and limitations.

\section{References}

[1] G. Caldiera and V. R. Basili. Identifying and qualifying reusable software components. Computer, 24:61-70, Febuary 1991.

[2] L. H. Etzkorn and C. G. Davis. Automatically identifying reusable oo legacy code. Computer, 30:66-71, October 1997.

[3] J. F. Girard and R. Koschke. Finding components in a hierarchy of modules: a step towards architectural understanding. In Proceedings of the 13th International Conference on Software Maintenance (ICSM'97), pages 58-65, Bari, Italy, October 1997.

[4] JESS, a rule engine for the java platform, 2005. http://www.jessrules.com/jess/index.shtml.

[5] Rich Client Platform, 2005. www.eclipse.org/rcp.

[6] S. K. Shin and S. D. Kim. A method to transform objectoriented design into component-based design using object-z. In Proceedings of the 3rd International Conference on Software Engineering Research, Management and Applications (SERA'05), pages 274-281, Michigan, USA, August 2005.

[7] The Java Parser Generator, 2005. https://javacc.dev.java.net.

[8] J. A. Wang. Towards component-based software engineering. Computing Sciences in Colleges, 16:177-189, October 2000. 